\title{
Quantization on Curves
}

\author{
CHRISTIAN FRØNSDAL \\ Department of Physics and Astronomy, University of California, Los Angeles, \\ CA 90095-1547, USA. e-mail: fronsdal@physics.ucla.edu
}

\section{With an Appendix by}

\author{
MAXIM KONTSEVICH \\ Institut de Hautes Etudes Scientifiques, 35 route de Chartres, 91440 Bures sur Yvette, \\ France.e-mail: maxim@ihes.fr
}

Received: 30 June 2002; appendix added: 7 June 2004; revised version: 12 September 2006 Published online: 19 January 2007

\begin{abstract}
Deformation quantization on varieties with singularities offers perspectives that are not found on manifolds. The Harrison component of Hochschild cohomology, vanishing on smooth manifolds, reflects information about singularities. The Harrison 2 -cochains are symmetric and are interpreted in terms of abelian $*$-products. This paper begins a study of abelian quantization on plane curves over $\mathbb{C}$, being algebraic varieties of the form $\mathbb{C}^{2} / R$, where $R$ is a polynomial in two variables; that is, abelian deformations of the coordinate algebra $\mathbb{C}[x, y] /(R)$. To understand the connection between the singularities of a variety and cohomology we determine the algebraic Hochschild (co)homology and its Barr-Gerstenhaber-Schack decomposition. Homology is the same for all plane curves $\mathbb{C}[x, y] / R$, but the cohomology depends on the local algebra of the singularity of $R$ at the origin. The Appendix, by Maxim Kontsevich, explains in modern mathematical language a way to calculate Hochschild and Harrison cohomology groups for algebras of functions on singular planar curves etc. based on Koszul resolutions.
\end{abstract}

Mathematics Subject Classification (2000). 53D55, 14A22, 16E40, 16S60, 81S10.

Keywords. quantization, deformation, Harrison cohomology, singular curves.

\section{Introduction}

Deformation quantization is a term coined by Moshe Flato, who suggested that any nontrivial associative deformation of an algebra of functions should be interpreted as a kind of "quantization". Deformation quantization is [3] the study of associative $*$-products of the form $f * g=f g+\sum_{n>0} \hbar^{n} C_{n}(f, g)$, where $\hbar$ is a formal parameter [11]. This concept has gained wide currency and has been intensively developed in recent years, but almost exclusively in the context of smooth Poisson manifolds ([4], [5, earlier papers] and [16,17]). In that case it is natural to consider deformations "in the direction of the Poisson bracket" (Drinfel'd); that 
is, taking $C_{1}(f, g)=\{f, g\}$, which is of course antisymmetric. But even if more general deformations were to be considered, independent of the symplectic structure, antisymmetry of $C_{1}$ entails no essential loss of generality for quantization on a smooth (finite-dimensional) manifold. A famous result of Hochschild, Kostant and Rosenberg [15] implies that any $*$-product on a regular, commutative algebra is equivalent to one with antisymmetric $C_{1}$. For a related "smooth" result, see [20].

It would seem, therefore, that the time has come to study deformation quantization on varieties with singularities. The cohomological implication of singularities should be interesting.

The Hochschild complex of any commutative algebra decomposes into smaller complexes; in the case of an algebra $A$ generated by $N$ generators, into $N$ subcomplexes $[1,2,6,13]$. The topology of a smooth manifold is related to the restriction of the Hochschild complex to alternating maps $A^{\wedge} \rightarrow A$, dual to simplicial homology, and the only component with non-vanishing cohomology. But on varieties with singularities other components of the Hochschild complex come into play, which suggests the use of cohomological methods for the study of singularities.

Examples of quantization on singular varieties had been known in connection with geometric quantization (and $*$-quantization) on coadjoint orbits of Lie algebras, but the cohomological implications had not been recognized; see [3,7-9]. The connection between singularities and cohomology was studied by Harrison [14], who was the first to describe the component of Hochschild cohomology that has become known, if not widely known, as Harrison cohomology. The 2-cochains of this complex are symmetric. On a commutative algebra every exact Hochschild 2-cochain is symmetric, so that triviality is not an issue if $C_{1}$ is antisymmetric. But it is an important consideration in the case of abelian $*$-products.

\subsection{THE BGS IDEMPOTENTS}

The $p$-chains of the Hochschild homology complex of a commutative algebra $A$ are the $p$-tuples $a=\sum a_{1} \otimes \cdots \otimes a_{p} \in A^{\otimes p}$, and the differential is defined by

$$
\mathrm{d} a=a_{1} a_{2} \otimes a_{3} \otimes \cdots \otimes a_{p}-a_{1} \otimes a_{2} a_{3} \otimes a_{4} \cdots \otimes a_{p}+\cdots+(-)^{p} a_{1} \otimes \cdots a_{p-2} \otimes a_{p-1} a_{p} .
$$

The $p$-cochains are maps $A^{\otimes p} \rightarrow A$, and the differential is

$$
\delta C\left(a_{1}, \ldots, a_{p+1}\right)=a_{1} C\left(a_{2}, \ldots, a_{p-1}\right)-C(\mathrm{~d} a)-(-)^{p} C\left(a_{1}, \ldots, a_{p}\right) a_{p+1} .
$$

After the pioneering work of Harrison [14] and Barr [1,2], the complete decomposition of the Hochschild cohomology of a commutative algebra was found by Gerstenhaber and Schack $[12,13]$. The Hochschild cochain complex splits into an infinite sum of direct summands. (If the algebra is generated by $N$ generators then there are only $N$ nonzero summands.) The decomposition is based on the action of $S_{n}$ on $n$-cochains, and on the existence of $n$ idempotents $e_{n}(k), k=1, \ldots, n$, in $\mathbb{C} S_{n}, \sum_{k} e_{n}(k)=1$, with the property that $\delta \circ e_{n}(k)=e_{n+1}(k) \circ \delta$. Thus we have $\operatorname{Hoch}_{n}=\sum_{k=1}^{n} H_{n, k}, \operatorname{Hoch}^{n}=\sum_{k=1}^{n} H^{n, k}$ with $H_{n, 1}=\operatorname{Harr}_{n}$ and $H^{n, 1}=\operatorname{Harr}^{n}$. 
A generating function was found by Garsia [10],

$$
\sum_{k=1}^{n} x^{k} e_{n}(k)=\frac{1}{n !} \sum_{\sigma \in S_{n}}\left(x-d_{\sigma}\right)\left(x-d_{\sigma}+1\right) \cdots\left(x-d_{\sigma}+n-1\right) \operatorname{sgn}(\sigma) \sigma
$$

where $d_{\sigma}$ is the number of descents, $\sigma(i)>\sigma(i+1)$, in $\sigma(1 \ldots n) .^{1}$ The simplest idempotents are

$$
\begin{aligned}
e_{2}(1) 12 & =\frac{1}{2}(12+21), \\
e_{3}(1) 123 & =\frac{1}{6}(2(123-321)+132-231+213-312), \\
e_{3}(2) 123 & =\frac{1}{2}(123+321) \\
e_{n}(n) & =\frac{1}{n !} \sum_{\sigma \in S_{n}} \operatorname{sgn}(\sigma) \sigma .
\end{aligned}
$$

The Hochschild chains decompose in the same way, with $d \circ e_{n}(k)=e_{n-1}(k) \circ d$.

\subsection{SUMMARY OF SECTIONS 2 TO 5}

Section 2 is concerned with abelian $*$-products on an arbitrary plane curve. The space of equivalence classes of first-order abelian deformations of the algebra of polynomials on $\mathbb{C}[x, y] /(R)$ is isomorphic to the local algebra of the singularity of $R$ at $x=y=0$. The Harrison component $\operatorname{Harr}^{3}=H^{3,1}$ of Hoch $^{3}$ vanishes, which implies that there are no obstructions to continuing a first-order abelian $*$-product to higher orders. In this paper the strategy that leads to the calculation of Hochschild cohomology calls for a preparatory investigation of a homological complex that is not strictly Hochschild, but rather its restriction $A \rightarrow A_{+}$to the non-unital subalgebra $A_{+}$of positive degree; this has no effect on the cohomology.

In Section 3 the Hochschild homology is calculated for the case of a plane curve, with its BGS decomposition. In Section 4 the Hochschild cohomology is investigated; the result in Theorem 4.9. Section 5 contains a detailed calculation of the BGS decomposition for the singularity of $x^{n}=0$ at $x=0$.

\section{Associative $*$-Products and Cohomology}

\subsection{FORMAL $*$-PRODUCTS}

A formal, abelian $*$-product on a commutative algebra $A$ is a commutative, associative product on the space of formal power series in a formal parameter $\hbar$ with

\footnotetext{
${ }^{1}$ Example: $\sigma(1234)=3142$ has one descent, from 2 to 3 .
} 
coefficients in $A$, given by a formal series

$$
f * g=f g+\sum_{n>0} \hbar^{n} C_{n}(f, g) .
$$

Associativity is the condition that $f *(g * h)=(f * g) * h$, or

$$
\left.\sum_{m, n=0}^{k} \hbar^{m+n}\left(C_{m}\left(f, C_{n}(g, h)\right)-C_{m}\left(C_{n}(f, g), h\right)\right)\right)=0,
$$

where $C_{0}(f, g)=f g$. This must be interpreted as an identity in $\hbar$; thus

$$
\left.\sum_{m, n=0}^{k} \delta_{m+n, k}\left(C_{m}\left(f, C_{n}(g, h)\right)-C_{m}\left(C_{n}(f, g), h\right)\right)\right)=0, \quad k=1,2, \ldots
$$

The formal $*$-product (2.1) is associative to order $p$ if Equation (2.3) holds for $k=1, \ldots, p$.

A first-order abelian $*$-product is a product

$$
f * g=f g+\hbar C_{1}(f, g), \quad C_{1}(f, g)=C_{1}(g, f),
$$

associative to first order in $\hbar$, which is the requirement that $C_{1}$ be closed,

$$
\delta C_{1}(f, g, h):=f C_{1}(g, h)-C_{1}(f g, h)+C_{1}(f, g h)-C_{1}(f, g) h=0 .
$$

Suppose that a formal $*$-product is associative to order $p \geqslant 1$; this statement involves $C_{1}, \ldots, C_{p}$ only, and we suppose these cochains fixed. Then the condition that must be satisfied by $C_{p+1}$, in order that the $*$-product be associative to order $p+1$, is

$$
\left.\sum_{\substack{m, n=1 \\ m+n=p+1}}^{p}\left(C_{m}\left(f, C_{n}(g, h)\right)-C_{m}\left(C_{n}(f, g), h\right)\right)\right)=-\delta C_{p+1}(f, g, h) .
$$

The left-hand side is closed, and thus it is seen that the obstructions to promote associativity from order $p$ to order $p+1$ are in $\mathrm{Hoch}^{3}$.

There is an important difference between the two cases of symmetric and antisymmetric $C_{1}$. If $C_{1}, \ldots, C_{p}$ are symmetric, then the left-hand side of (2.5) has the symmetry of the idempotent $e_{3}(1)$ (a Harrison cochain) and it is the symmetric part of $C_{p+1}$ that is relevant, while the antisymmetric part of $C_{p+1}$ must simply be closed. Symmetry of the $*$-product can therefore be maintained to all orders. If $C_{1}$ is antisymmetric, and $p=1$, then the left-hand side has the symmetry of $e_{3}(1)+e_{3}(3)$. The first part must be balanced on the right-hand side by means of the symmetric part of $C_{2}$; the second part must vanish, and this condition is the Jacobi identity for $C_{1}$.

The obstructions against continuing a formal, first order, abelian $*$-product to higher orders are in $\operatorname{Hoch}^{3}$; more precisely, they are in $H^{3,1}=\operatorname{Harr}^{3}(A, A)$. 
A formal $*$-product is trivial if there is an invertible map $E: A \rightarrow A$, in the form of a formal series $E(f)=f+\sum_{n>0} \hbar^{n} E_{n}(f)$ such that $E(f * g)=E(f) E(g)$. A first order, abelian $*$-product is trivial if there is a 1-cochain $E_{1}$ such that

$$
C_{1}(f, g)=\delta E_{1}(f, g)=f E_{1}(g)-E_{1}(f g)+E_{1}(f) g .
$$

\subsection{DEFORMATIONS ON A CURVE}

In view of the theorem of Hochschild, Kostant and Rosenberg [15] cited earlier, there can be no nontrivial, abelian $*$-products on a smooth manifold. It is natural to turn to varieties with singularities, and especially algebraic varieties. It is the aim of this paper to explore the phenomena, with elementary methods of calculation, in the case of plane curves over $\mathbb{C}, M=\mathbb{C}^{2} / R$, where $R$ is a $\mathbb{C}$-polynomial. The algebras of interest are the coordinate algebra

$$
A=\mathbb{C}[x, y] /(R),
$$

with generators $x, y$ and a single polynomial relation $R$. The polynomial $R$ can be transformed, by a linear change of variables, to either of the forms $R=x^{m}-$ $P(x, y)$ or $R=y^{n}-Q(x, y)$, where the polynomial $P$ is of order less than $m$ in $x$ and the polynomial $Q$ is of order less than $n$ in $y$. Either form gives rise to a Poincaré-Witt basis for $A$, for example, $x^{i} y^{j}, i=0,1, \ldots, \infty, j=0,1, \ldots, n-1$.

The deformed algebra has a Poincaré-Witt basis of the same form. Let $W$ be the map that takes a $*$-monomial of this basis to the same ordinary monomial of the original basis. Let $R_{\hbar}:=W\left(R^{*}\right)$ and let $M_{\hbar}:=\mathbb{C}^{2} / R_{\hbar}$. Then, morally, the *-product is trivial if there is a bijection $E: M_{\hbar} \rightarrow M$ such that $R_{\hbar} \mapsto R$. However, since $\hbar$ is a formal parameter, the following definition is preferred.

DEFINITION 2.3. A $*$-product, as defined in this section, is trivial if there is a mapping by a formal power series in $\hbar, E=\mathrm{Id}+\sum_{n>1} \hbar^{n} E_{n}$, such that $R_{\hbar} \mapsto R$.

\subsection{FIRST-ORDER $*$-PRODUCT ON A CURVE}

Consider a first-order, associative and abelian $*$-product on the algebra (2.6), with the polynomial $R$ in the form $R=y^{n}-Q(x, y)$. A change of variables ensures that $\left(x^{i} y^{j}\right) *\left(x^{k} y^{l}\right)=x^{i+k} y^{j+l}$ for $j+l<n$ and

$$
y^{i} * y^{n-i}=Q(x, y)+\hbar Q_{1}(x, y), \quad 1 \leqslant i \leqslant n-1,
$$

The first-order deformation (2.7) is trivial if there is a derivation $E$ such that $Q_{1}=E(R)$. See Subsection 4.6.

Example 2.5. Let $A=\mathbb{R}[x, y] /(R), R=y^{2}-x^{2}-r^{2}, r^{2} \in \mathbb{C}$, decompose $f \in A$ as $f=$ $f_{+}+y f_{-}, f_{ \pm} \in \mathbb{R}[x]$, and define a $*$-product on $A$ by setting $f * g=f g+\hbar f_{-} g_{-}$. 
Then $Q_{1}=1$ and we seek $E$ such that $E\left(x^{2}+r^{2}-y^{2}\right)=1$. The general solution to this equation is $2 E=\frac{-1}{r^{2}}\left(x \partial_{x}+y \partial_{y}\right)+\alpha\left(y \partial_{x}+x \partial_{y}\right)$, with $\alpha \in A$.

Of course, this breaks down if $r^{2}=0$, and the simple reason why there is no solution in this case is that there is no differential operator $E$ such that the polynomial $E\left(x^{2}-y^{2}\right)$ contains a constant term.

PROPOSITION 2.6. Let $X$ be the space of polynomials in $x$ and $y$, of degree less than $n$ in $y$, and let $D R$ be the gradient ideal of $R$. As vector spaces, $X$ coincides with $A$ and $D R$ consists of all differentials of $R$. The space of equivalence classes of essential, first-order $*$-products on $A$ is the space $X / D R, \operatorname{Harr}^{2}(A, A)=X / D R$.

Example 2.7. Let $M=\mathbb{C}^{2}, R=y^{2}-x^{3}$. A full set of representatives of $X / D R$ is $a+b x, a, b \in \mathbb{C}$. The deformed algebras are $A_{\hbar}=\mathbb{C}[x, y] / R_{\hbar}$ with $R_{\hbar}=y^{2}-x^{3}-$ $\hbar(a x+b)$. Expand $f(x, y)=f_{+}(x)+y f_{-}(x)$. Then $f * g=f g+\hbar C_{1}(f, g)$, where $C_{1}(f, g)=(a x+b) f_{-} g_{-}$.

\section{Homology}

This section deals with the homology of a modified Hochschild complex. The strategy that is used in this paper, to calculate the Hochschild cohomology of $A$, begins by a determination of the homology of the algebra $A_{+}$, the subalgebra with positive degree of $A$. The $n$-chains of this homology of $A_{+}$are $n$-tuples $a=a_{1} \otimes a_{2} \otimes \cdots a_{n}, a_{i} \in A_{+}, i=1, \ldots, N$.

\subsection{2-CHAINS}

Every "Hochschild" 2-chain is homologous to a 2-chain of the form $x \otimes a+y \otimes b$. It will be convenient to relabel the generators, $x, y \mapsto x_{1}, x_{2}$, then $a \approx \sum x_{i} \otimes a_{i}$, $a_{i} \in A_{+}, i=1,2$. It is closed if $\sum x_{i} a_{i}=0$. We shall suppose that $R$ has no constant term and no linear terms, then $a$ has the representation

$$
a \approx \sum x_{i} \otimes x_{j} \varepsilon^{i j} b+\sum_{i=1}^{2} x_{i} \otimes R_{i} c,
$$

where $\varepsilon^{i j}=-\varepsilon^{j i}, \varepsilon^{12}=1, \quad \sum x_{i} R_{i}=R$ and where $b, c$ are in the unital augmentation $A$ of $A_{+}$. The first term is exact if $b \in A_{+}$, the second term is exact if $c \in$ $A_{+}$and (a section of) $H_{2}=Z_{2} / B_{2}$ is spanned (over $\mathbb{C}$ ) by the chains $x_{1} \wedge x_{2}$ and $\sum x_{i} \otimes R_{i}$. The second one is homologous to a symmetric chain that is a basis for $\operatorname{Harr}_{2}=H_{2,1}$.

Example 3.2. If $R=y^{2}-x^{n}$, then $\mathrm{Harr}_{2}$ has dimension 1 and every symmetric, closed 2-chain is homologous to a $\mathbb{C}$-multiple of $x \otimes x^{n-1}+x^{n-1} \otimes x-2 y \otimes y$. 


\subsection{3-CHAINS}

Every 3-chain is homologous to one of the form $a=\sum x_{i} \otimes b_{j} \otimes c^{i j}$. If $a$ is closed it takes the form $a \approx \sum x_{i} \otimes x_{j} \varepsilon^{i j} b \otimes b^{\prime}+x_{i} \otimes R_{i} c \otimes c^{\prime}, \quad b, c \in A$ which is homologous to $a \approx \sum x_{i} \otimes x_{j} \varepsilon^{i j} \otimes b b^{\prime}+x_{i} \otimes R_{i} \otimes c c^{\prime}$, with $x_{2} b b^{\prime}+R_{1} c c^{\prime}=0$ and $-x_{1} b b^{\prime}+R_{2} c c^{\prime}=0$. A simple case-by-case study shows that we then have:

$$
b b^{\prime}=\alpha R_{1}+\beta R_{2}, \quad c c^{\prime}=-\alpha x_{2}+\beta x_{1},
$$

with $\alpha, \beta$ in $A$. Thus any closed 3-chain is homologous to one of the form

$$
\left(\left(x_{1} \wedge x_{2}\right) \otimes R_{1} c_{1}-\sum x_{i} \otimes R_{i} \otimes x_{2} c_{1}\right)-\left(\left(x_{1} \wedge x_{2}\right) \otimes R_{2} c_{2}+\sum x_{i} \otimes R_{i} \otimes x_{1} c_{2}\right) .
$$

The first (second) term is exact unless $c_{1}\left(c_{2}\right)$ is in $\mathbb{C}$. Adding an exact, alternating 3 -cycle we get an alternative section of $Z_{3} / B_{3}$ with a basis that consists of the two chains (the GS idempotents were defined in Section 1)

$$
\begin{aligned}
& \alpha_{1}=e_{3}(2)\left(x_{1} \otimes x_{2} \otimes R_{1}-x_{2} \otimes R_{1} \otimes x_{1}-x_{2} \otimes x_{1} \otimes R_{1}-x_{2} \otimes R_{2} \otimes x_{2}\right), \\
& \alpha_{2}=e_{3}(2)\left(x_{2} \otimes x_{1} \otimes R_{2}-x_{1} \otimes R_{2} \otimes x_{2}-x_{1} \otimes x_{2} \otimes R_{2}-x_{1} \otimes R_{1} \otimes x_{1}\right) .
\end{aligned}
$$

Thus $\mathrm{Hoch}_{3}=H_{3,2}$ has dimension 2 and $\mathrm{Harr}_{3}=0$.

Another way to reach this conclusion is to differentiate (3.1). The result is $\left(c_{1} x_{2}+c_{2} x_{1}\right) \wedge R$, which is in $Z_{2,2}$ and which implies that $(3.1) \in Z_{3,2}$.

Example 3.4. If $R=y^{2}-x^{2}$, set $u=x+y, v=x-y$. The basis (3.2) is then $\{u \otimes v\} \otimes u, v \otimes\{u \otimes v\}$ and the dimension of $\mathrm{Hoch}_{3}$ is 2. More precisely, dim $H_{3, k}$ is $0,2,0$ for $k=1,2,3$.

Example 3.5. If $R=y^{2}-x^{3}$, then the chains (3.2) become

$$
y \otimes x \otimes y-x \otimes y \otimes y-y \otimes y \otimes x+x \otimes x^{2} \otimes x
$$

and

$$
e_{3}(2)\left(x \otimes y \otimes x^{2}-y \otimes x^{2} \otimes x-x^{2} \otimes x \otimes y+y \otimes y \otimes y\right) .
$$

It is straightforward to prove the following.

PROPOSITION 3.6. Let $P^{1}=\left\{x_{1}, x_{2}\right\}, P^{n+1}=P^{n} \otimes M_{n}$, and

$$
M_{2 k+1}=\left(\begin{array}{cc}
R_{1} & -x_{2} \\
R_{2} & x_{1}
\end{array}\right), \quad M_{2 k}=\left(\begin{array}{cc}
x_{1} & x_{2} \\
-R_{2} & R_{1}
\end{array}\right) .
$$

Then for $n>1$ every closed $n$-chain is homologous to an $n$-chain in the linear span of the two linearly independent polynomials in $P^{n}$. 
Example 3.7. If $R=y^{2}-x^{2}$, set $u=x+y, v=x-y$. The dimension of $\mathrm{Hoch}_{n}$ is 2 ; the basis is $\{u \otimes v \otimes u \cdots, \quad v \otimes u \otimes v \otimes u \cdots\}$.

THEOREM 3.8. Hoch H $_{k}=H_{2 k, k}+H_{2 k, k+1}$, each component one-dimensional over $\mathbb{C}$, and Hoch $_{2 k-1}=H_{2 k-1, k}$, two-dimensional over $\mathbb{C}, k=1,2, \ldots$

Proof. For $k=1, \ldots, p-1, P^{p+1}=P^{k} \otimes M_{k} \otimes M_{k+1} \otimes \cdots \otimes M_{p}$ and thus

$$
\mathrm{d} P^{p+1}=P^{1} M_{1} \otimes M_{2} \otimes \cdots \otimes M_{p}+\sum_{k=1}^{p-1}(-)^{k} P^{k} \otimes M_{k} M_{k+1} \otimes \cdots \otimes M_{p} .
$$

We have $M_{k} M_{k+1}=R$ times the unit matrix and $P^{1} M_{1} \otimes M_{2}=R \otimes P^{1}$; consequently $\mathrm{d} P^{1}=0, \mathrm{~d} P^{2}=\{R, 0\}$ and $\mathrm{d} P^{p+1}=R(s h) P^{p-1}, \quad p \geqslant 2$. If $a \in C_{p, k}$, then $\mathrm{d} a \in$ $C_{p-1, k}$, and $R(s h) a$ is homologous to some $b \in C_{p+1, k+1}$. Hence if $P^{p-1} \in C_{p-1, k}$, then $P^{p+1}$ is homologous to a $C_{p+1, k+1}$ chain. The action of these maps between spaces with cohomology is shown in the diagram.

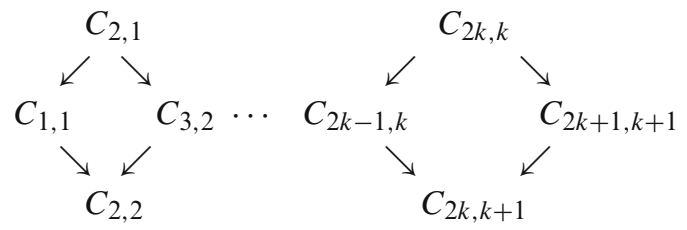

A southeast arrow represents the map $a \mapsto R(s h) a$; a southwest arrow is the action of the differential. The projections of $\left\{P_{i}^{2 k+1}\right\}_{i=1,2}$ form a basis for $H_{2 k+1, k+1}$ and the projections of $P_{1}^{2 k}$ (respectively $P_{2}^{2 k}$ ) are bases for $H_{2 k, k}$ (respectively $H_{2 k, k+1}$ ).

\section{Cohomology}

\subsection{THE REDUCTION PROCESS}

The chains considered in this section are restricted to positive degree. The cochains are valued in $A$. A $p$-cochain is closed if

$$
\delta C\left(a_{1}, \ldots, a_{p+1}\right)=a_{1} C\left(a_{2}, \ldots, a_{p+1}\right)-C(d a)-(-)^{p} C\left(a_{1}, \ldots, a_{p}\right) a_{p+1}=0 .
$$

One may attempt to interpret this relation as fixing the value $C(\mathrm{~d} a)$, recursively in the degree of the argument. The obstruction to this is $\mathrm{d} a=0$, but if $a$ is exact then (4.1) is satisfied automatically by virtue of its being true for arguments of lower degree. (One can show that, in this context, if $a$ is exact then there is $b$ of the same degree such that $a=\mathrm{d} b$.) It is enough, therefore, to verify closure for a basis of representatives of $\operatorname{Hoch}_{p+1}$.

A closed $p$-cochain $C$ is a coboundary if there is a $(p-1)$-cochain $E$ such that

$$
C(a)=a_{1} E\left(a_{2}, \ldots, a_{p}\right)-E(\mathrm{~d} a)+(-)^{p} E\left(a_{1}, \ldots, a_{p-1}\right) a_{p} .
$$


This relation can be solved for $E(\mathrm{~d} a)$, recursively by increasing degree, except for the obstruction presented by $\mathrm{d} a=0$. But if $a=\mathrm{d} b$ then $C(a)$ is determined by $\delta C(b)=0$. So it is enough to examine (4.2) for a complete set of representatives of $\operatorname{Hoch}_{p}$.

The most useful interpretation is this. Given any closed $p$-cochain a "gauge transformation" is the addition of an exact $p$-cochain, $C \rightarrow C+\Delta C$, with

$$
\Delta C\left(a_{1} \ldots a_{p}\right)=a_{1} E\left(a_{-}\right)+(-)^{p} a_{p} E\left(a_{+}\right)-E(\mathrm{~d} a) .
$$

The space $\operatorname{Hoch}^{p}$ is the spac of closed, gauge-invariant $p$-cochains.

If any BGS component $H_{p, k}$ of Hoch ${ }_{p}$ vanishes then the corresponding component $H^{p, k}$ of Hoch ${ }^{p}$ is zero. There are no obstructions to continuing a first-order, abelian *-product to higher orders.

\subsection{CLOSURE FOR $p=1$}

The 2-homology is spanned by $x_{1} \wedge x_{2}$ and $x_{i} \otimes R_{i}$. We shall replace the latter by $\hat{R}=\sum A_{i j} x_{1}^{i} \otimes x_{2}^{j}, \quad R=\sum A_{i j} x_{1}^{i} x_{2}^{j}$. The relation $\delta C\left(x_{1} \wedge x_{2}\right)=0$ is trivial. The formula $\delta C\left(x_{1}^{i} \otimes x_{2}^{j}\right)=x_{1}^{i} C\left(x_{2}^{j}\right)+x_{2}^{j} C\left(x_{1}^{i}\right)-C\left(x_{1}^{i} x_{2}^{j}\right)$ tells us that, if $C$ is closed, then for any polynomial $f, C(f)=C\left(x_{i}\right) \partial_{i} f$. Hence (this is the result 2.6)

$$
\delta C\left(P_{1}^{2}\right)=C\left(x_{i}\right) \partial_{i} R, \quad \delta C\left(P_{2}^{2}\right)=0 .
$$

For the algebra $\mathbb{C}[x, y], Z^{1}$ is the space of vector fields with coefficients in the unital augmentation of the same algebra, but for $A=\mathbb{C}[x, y] / R, Z^{1}$ is the algebra of vector fields that annihilate $R$ (the algebra of vector fields tangential to the curve).

\subsection{CLOSURE FOR $p=2$}

For homology we use the basis (3.3); it is enough to examine one of the two,

$$
\begin{aligned}
P_{1}^{3} & =\hat{R} \otimes x_{1}+x_{1} \wedge x_{2} \otimes R_{2}, \\
\delta C\left(P_{1}^{3}\right) & =x_{1} C\left(R_{1} \wedge x_{1}\right)+x_{2} C\left(R_{2} \wedge x_{1}\right)-R_{2} C\left(x_{1} \wedge x_{2}\right) .
\end{aligned}
$$

The first two arguments are exact; a certain amount of calculation is needed to verify that these terms are of the same form as the third one. We need the following simple formula, satisfied by closed 2-cochains: $C\left(x_{2} \wedge f\right)=C\left(x_{2} \wedge x_{1}\right) \partial_{1} f, f \in$ $A$. Now it follows easily that $\delta C\left(P_{1}^{3}\right)=-C\left(x_{1} \wedge x_{2}\right) \partial_{2} R, \delta C\left(P_{2}^{3}\right)=C\left(x_{1} \wedge x_{2}\right) \partial_{1} R$. Therefore, we can interpret the condition $\delta C(a)=0$ as fixing the value $C(\mathrm{~d} a)$, provided only that $C\left(P_{2}^{2}\right) \partial_{i} R=0, i=1,2$. (That is satisfied if $R=x^{2} y^{3}, C(x \wedge y)=x y$.)

THEOREM 4.4. Closure of a p-cochain $C$ implies that its values for exact arguments are given recursively in the polynomial degree as in (4.1). Conversely, (4.1) can 
be solved recursively for all $C(\mathrm{~d} a)$, if and only if the following conditions hold

$$
\begin{aligned}
C \in Z^{2 k, k+1}: C\left(P_{2}^{2 k}\right) \partial_{i} R=0, \quad i=1,2 ; \\
C \in Z^{2 k+1, k+1}: \sum C\left(P_{i}^{2 k+1}\right) \partial_{i} R=0 ; \\
C \in Z^{2 k, k}: \text { always. }
\end{aligned}
$$

\subsection{GAUGE INVARIANCE FOR $p=1$}

Trivial, all 1-cochains are gauge invariant, $H^{1}=Z^{1}$.

\subsection{GAUGE INVARIANCE FOR $p=2$}

We must examine evaluations on the homology basis. To begin with, $\Delta C\left(x_{1} \wedge\right.$ $\left.x_{2}\right)=0$, so that the evaluation $C\left(x_{1} \wedge x_{2}\right)$ is gauge invariant. To examine the supplementary homology space, set $R=\sum A_{i j} x_{1}^{i} x_{2}^{j}, \hat{R}=\sum A_{i j} x_{1}^{i} \otimes x_{2}^{j}$. Then we have

$$
\begin{gathered}
\sum_{2}^{1} A_{i j}\left(\Delta C\left(x_{1}^{i} \otimes x_{2}^{j}\right)+x_{1}^{i} \sum_{k=2}^{j-2} x_{2}^{k} \Delta C\left(x_{2} \otimes x_{2}^{j-1-k}\right)+\right. \\
\left.+x_{2}^{j} \sum_{k=0}^{i-2} \Delta C\left(x_{1} \otimes x_{1}^{i-k-1}\right)\right)=E\left(x_{i}\right) \partial_{i} R
\end{gathered}
$$

Hence, in a gauge where $C$ vanishes on arguments of lower degrees, $\Delta C(\hat{R}) \in$ $D R$ and we have recovered Proposition 2.6.

\subsection{GAUGE INVARIANCE FOR $p=3$}

We have

$$
\begin{aligned}
\delta C\left(P_{1}^{3}\right) & =\Delta C\left(\hat{R} \otimes x_{1}+x_{1} \wedge x_{2} \otimes R_{2}\right)= \\
& \left.=x_{1} E\left(R_{1} \wedge x_{1}\right)+x_{2} E\left(R_{2} \wedge x_{1}\right)\right)-R_{2} E\left(x_{1} \wedge x_{2}\right)= \\
& =\sum \frac{1}{2} A_{i j}\left\{x_{1} E\left(x_{1}^{i-1} x_{2}^{j} \wedge x_{1}\right)+x_{2} E\left(x_{1}^{i} x_{2}^{j-1} \wedge x_{1}\right)\right\}-R_{2} E\left(x_{1} \wedge x_{2}\right) .
\end{aligned}
$$

With the help of the identity

$$
\sum_{k=1}^{i-1} x_{1}^{k} \Delta C\left(x_{1} \otimes x_{1}^{i-k-1} x_{2}^{j} \otimes x_{1}\right)=x_{1}^{i} E\left(x_{2}^{j} \wedge x_{1}\right)-x_{1} E\left(x_{1}^{i-1} x_{2}^{j} \wedge x_{1}\right), j \geqslant 1,
$$


and another one, similar, we can reduce (4.5) to

$$
\begin{aligned}
& \Delta C\left(P_{1}^{3}\right)+\sum_{k=1}^{i-1} A_{i j} x_{1}^{k} \Delta C\left(x_{1} \otimes x_{1}^{i-k-1} x_{2}^{j} \otimes x_{1}\right)+ \\
& \quad+\sum_{k=1}^{i} A_{i j} x_{2} x_{1}^{k-1} \Delta C\left(x_{1} \otimes x_{1}^{i-k} x_{2}^{j-1} \otimes x_{1}\right)= \\
& =\sum A_{i j}\left\{x_{1}^{i} E\left(x_{2}^{j} \wedge x_{1}\right)+x_{1}^{i} E\left(x_{2}^{j-1} \wedge x_{1}\right)\right\}-R_{2} E\left(x_{1} \wedge x_{2}\right) .
\end{aligned}
$$

A similar, further reduction leads to the result that, if $\delta C$ vanishes on arguments of lower orders, $\Delta C\left(P_{1}^{3}\right)+\cdots=-\left(\partial_{2} R\right) E\left(x_{1} \wedge x_{2}\right), \Delta C\left(P_{2}^{3}\right)+\cdots=\left(\partial_{1} R\right) E\left(x_{1} \wedge x_{2}\right)$. We recall that $\Delta C(a)=\delta E\left(a_{1}\right)$ and remember from Subsection 4.3 that $\delta E=0$ implies that $\partial_{i} R E\left(x_{1} \wedge x_{2}\right)=0$. The above result is thus natural; the calculation is needed only to fix the numerical coefficients.

PROPOSITION 4.8. If the gauge is fixed by the condition that $C(a)=0$ for arguments a of lower degree, then the remaining gauge transformations take the following form,

$$
\begin{aligned}
\Delta C\left(P^{1}\right) & =0, \quad \Delta C\left(P_{1}^{2 k}\right)=\sum E_{i} \partial_{i} R, \\
\Delta C\left(P_{2}^{2 k}\right) & =0, \quad \Delta C\left(P^{2 k+1}\right)=E d R^{*}, \quad k>0 .
\end{aligned}
$$

Proof. (outline). (a) The statement reflects the structure of (3.4). The dimension of $H^{p, k}$, over the local algebra, more or less, coincides with the dimension of $H_{p, k}$. "More or less" comes from the existence of homologies of lower orders, as the complete calculation in Subsection 4.7 shows.

(b) We have

$$
\begin{aligned}
\Delta C\left(P_{1}^{2 k}\right) & =\sum R_{i} E\left(P_{i}^{2 k-1}\right)+\sum x_{i} E\left(Q_{i}\right)+\cdots, \\
\Delta C\left(P_{i}^{2 k+1}\right) & =\sum \epsilon_{i j} R_{j} E\left(P_{2}^{2 k-1}\right)+\sum x_{j} E\left(S_{i j}\right), \\
\Delta C\left(P_{2}^{2 k}\right) & =\sum x_{i} E\left(T_{i}\right) .
\end{aligned}
$$

The chains $Q_{i}, S_{i j}, T_{i}$ are closed and, unless $R_{1}$ or $R_{2}$ is linear, exact. The reduction exemplified in (4.4) and in (4.5) is then available. The result is $\Delta C\left(P_{1}^{2 k}\right)+\cdots=E\left(P_{i}^{2 k-1}\right) \partial_{i} R, \quad \Delta C\left(P_{i}^{2 k+1}\right)+\cdots=E\left(P_{2}^{2 k-1}\right) \varepsilon_{i j} \partial_{j} R, \quad \Delta C\left(P_{2}^{2 k}\right)=0$.

(c) The last case $\left(P_{2}^{2 k} \in C_{2 k, k+1}\right)$ is simpler than the others and we give the details in that case only. Let $\tau \in S_{p}$ be the reversing permutation. Garsia's formula tells us that the chains $C_{p, k}$ correspond to the character $\tau \mapsto(-)^{k}$, so the projection $e_{2 k}(k+1) P_{2}^{2 k}$ has $\tau \mapsto(-)^{k+1}$. Now $\Delta C\left(P_{2}^{2 k}\right)=\sum_{i=1}^{2} x_{i} E\left(a_{i}\right)$, with $a_{i} \in C_{2 k-1}$ closed and with the same symmetry: $\tau \mapsto(-)^{k+1}$. The symmetry of $C_{2 k-1, k}$ (where the homology is) is $(-)^{k}$; therefore $a_{1}$ and $a_{2}$ are exact. The reduction process encounters no homology and leads to zero. 
Putting it all together we get the following result (for notations, see Propositions 2.6 and 4.8).

THEOREM 4.9. Let $V_{R}$ be the space of vector fields, with values in A, that annihilate $R$. Then as vector spaces,

$$
\begin{aligned}
H^{1} & =V_{R}, \\
H^{2 k, k} & =A / D R, \\
H^{2 k, k+1} & =\left\{a \in A_{+}, a \partial_{1} R=a \partial_{2} R=0\right\}, \\
H^{2 k+1, k+1} & =V_{R} /\left\{A \mathrm{~d} R^{*}\right\}, \quad k>0 .
\end{aligned}
$$

\section{Deformation of $x^{n}=0$}

Here, we complete the calculation of Hochschild cohomology of the algebra $A=$ $\mathbb{C}[x] / x^{n}, n \geqslant 2$. This purely algebraic problem, though not associated with a curve, is nevertheless very similar to that posed by curves. In the context of singularity theory it is one of the standard forms. The chains are restricted to positive degree. This subalgebra of $A$ is denoted $A_{+}$.

\subsection{HOMOLOGY}

For convenience, $x x x^{2} \ldots$ shall stand for either $x \otimes x \otimes x^{2} \ldots$ or $x, x, x^{2}, \ldots$ The spaces $H_{p}$ are one-dimensional for $p \geqslant 1$ and representative elements of $Z_{p}$ are $x$, $x x^{n-1}, x x^{n-1} x, \ldots$, or $\left(x x^{n-1}\right)^{k}$ for $p=2 k$ and $\left(x x^{n-1}\right)^{k} x$ for $p=2 k+1$.

\subsection{CLOSED COCHAINS}

A $p$-cochain $C$ is closed if

$$
\delta C\left(a_{1} \ldots a_{p+1}\right):=a_{1} C\left(a_{-}\right)+(-)^{p+1} a_{p+1} C\left(a_{+}\right)-C(\mathrm{~d} a)=0
$$

with $a_{-}=a_{2} \ldots a_{p+1}, a_{+}=a_{1} \ldots a_{p}$. We interpret this relation, in the first place, as a recursion relation that determines the cochain $C$ on exact arguments, in terms of its values on arguments of lower degree. For example, if the 1-cochain $C$ is closed, then $C\left(x^{k}\right)=k x^{k-1} C(x), k=2, \ldots, n$. Hence $C\left(x^{k}\right)$ is determined for $k=2, \ldots, n-1$ by $C(x)$, and $C(x) \in A_{+}$(thus restricted to positive degree).

The obstruction to this interpretation of (5.1) is $\mathrm{d} a=0$; in this case closure requires the relation

$$
\delta C(a)=a_{1} C\left(a_{-}\right)+(-)^{p+1} a_{p+1} C\left(a_{+}\right)=0 .
$$


But if $a=\mathrm{d} b$, then this last relation is automatic, since

$$
\begin{aligned}
\delta C(\mathrm{~d} b)= & b_{1} b_{2} C\left(b_{3} \ldots\right)-b_{1} C\left(\mathrm{~d} b_{-}\right)+ \\
& +(-)^{p+1} b_{p+2} C\left(\mathrm{~d} b_{+}\right)-b_{p+1} b_{p+2} C\left(b_{1} \ldots b_{p}\right) \\
= & b_{1} b_{2} C\left(b_{3} \ldots\right)-b_{1}\left(b_{2} C\left(b_{3} \ldots\right)+(-)^{p+1} b_{p+2} C\left(b_{2} \ldots b_{p+1}\right)\right) \\
& -b_{p+1} b_{p+2} C\left(b_{1} \ldots b_{p}\right) \\
& +b_{p+2}\left((-)^{p+1} b_{1} C\left(b_{2} \ldots b_{p+1}\right)+b_{p+1} C\left(b_{1} \ldots b_{p}\right)\right) \\
= & 0 .
\end{aligned}
$$

The real obstruction is thus the presence of homology. When $a=x x^{n-1} x \ldots$, then (5.2) reduces to

$$
\begin{aligned}
& p=2 k: x C\left(x^{n-1} \ldots x-x \ldots x^{n-1}\right)=0, \\
& p=2 k-1: x C\left(x^{n-1} \ldots x^{n-1}\right)+x^{n-1} C(x \ldots x)=0 .
\end{aligned}
$$

PROPOSITION 5.3. The obstructions to interpreting the closure condition (5.1) as recursively fixing the value of $C(\mathrm{~d} a)$ in terms of values of $C$ on arguments of lower degrees are: ${ }^{2}$

$$
p=2 k: \text { none, } \quad p=2 k-1: x^{n-1} C(x, \ldots, x) .
$$

Homology selects the argument here also. The truth of the Proposition is obvious except for the possibility of accidental cancellations. Here, nevertheless, is a direct proof.

Proof of Proposition 5.3. (case $p=2 k$ ). For $p=2 k$ and $m=1,2, \ldots, \alpha, \alpha=$ $k(n-2)+1$, let

$$
\phi^{m}:=\sum_{\substack{1 \leqslant p_{1}, \ldots, p_{k} \leqslant m \\ p_{1}+\cdots+p_{k}=k+m-1}} x x^{p_{1}} x x^{p_{2}} \ldots x x^{p_{k}} x .
$$

It may be shown by induction that

$$
\mathrm{d} \phi^{\alpha-1}=x^{n-1} \ldots x-x \ldots x^{n-1}, \quad \mathrm{~d} \phi^{m}=\phi_{-}^{m+1}-\phi_{+}^{m+1}, \quad m<\alpha-1 .
$$

Posing $\delta C\left(\phi^{m}\right)=0$ for $m<\alpha$, we find that the left-hand side of (5.3) vanishes identically:

$$
\begin{aligned}
x C\left(x^{n-1} \ldots x-x \ldots x^{n-1}\right) & =x C\left(\phi_{-}^{\alpha}-\phi_{+}^{\alpha}\right)=x C\left(d \phi^{\alpha-1}\right) \\
& =x^{2} C\left(\phi_{-}^{\alpha-1}-\phi_{+}^{\alpha-1}\right)=\cdots
\end{aligned}
$$

Iteration ends with $x^{n} C\left(a_{-}^{\alpha+1-n}-a_{+}^{\alpha+1-n}\right)=0$.

\footnotetext{
${ }^{2}$ From now on dots indicate a sequence in which $x$ and $x^{n-1}$ alternate.
} 
Proof of Proposition 5.3. (case $p=2 k-1$ ). For $m=1,2, \ldots, \alpha=k(n-2)+1$, set

$$
\psi^{m}:=\sum_{\substack{1 \leqslant p_{1}, \ldots, p_{k} \leqslant m \\ p_{1}+\cdots+p_{k}=k+m-1}} x x^{p_{1}} x x^{p_{2}} \ldots x x^{p_{k}} .
$$

Then $\mathrm{d} \psi^{\alpha-1}=x^{n-1} \ldots x^{n-1}=\psi_{-}^{\alpha}$ and for $m<\alpha-1, \quad \mathrm{~d} \psi^{m}=\psi_{-}^{m+1}-\phi^{m+1}$, and

$$
\left(x^{l} \psi_{p+1}^{\alpha-l}\right) \otimes \psi_{+}^{\alpha-l}=x^{n-1} \otimes \phi^{\alpha+2-n}, \quad l=0,1, \ldots n-2 .
$$

If $\delta C\left(\psi^{m}\right)=0, \quad m<\alpha$, then the left-hand side of (5.4) is

$$
\begin{array}{rl}
x & C\left(x^{n-1} \ldots x^{n-1}\right)+x^{n-1} C(x \ldots x) \\
& =x C\left(\mathrm{~d} \psi^{\alpha-1}+x^{n-1} C\left(\phi^{\alpha+2-n}\right)\right. \\
& =x^{2} C\left(\psi_{-}^{\alpha-1}\right)+2 x^{n-1} C\left(\phi^{\alpha+2-n}\right)=\ldots \\
& =x^{n-1} C\left(\psi_{-}^{\alpha+2-n}\right)+(n-1) x^{n-1} C\left(\phi^{\alpha+2-n}\right) \\
& =x^{n-1} C\left(\mathrm{~d} \psi^{\alpha+1-n}+\phi^{\alpha+2 n}\right)+(n-1) x^{n-1} C\left(\phi^{\alpha+2-n}\right) \\
& =n x^{n-1} C(x \ldots x) .
\end{array}
$$

The proof of Proposition 5.3 is complete. The implication is that, if a $(2 k-1)$ cochain $C$ is closed, then $C(x \ldots x) \in A_{+}$.

\subsection{EXACT COCHAINS}

Exact $p$-cochains have the form

$$
C\left(a_{1} \ldots a_{p}\right)=a_{1} E\left(a_{-}\right)+(-)^{p} a_{p} E\left(a_{+}\right)-E(\mathrm{~d} a) .
$$

The obstruction to interpreting this relation as a recursion relation to determine the $E(\mathrm{~d} a)$ is $\mathrm{d} a=0$. Here too, the real obstruction, when $C$ is closed, is the existence of homology. The most useful interpretation is this. Given any closed $p$ cochain a "gauge transformation" is the addition of an exact $p$-cochain, $C \rightarrow C+$ $\Delta C$, with

$$
\Delta C\left(a_{1} \ldots a_{p}\right)=a_{1} E\left(a_{-}\right)+(-)^{p} a_{p} E\left(a_{+}\right)-E(\mathrm{~d} a) .
$$

The space $H^{p}$ is the space of gauge invariant evaluations of closed $p$-cochains.

To illustrate, here is the situation for 2-cochains, when $n=3$. Closure,

$$
\delta C(x x x)=C\left(x x^{2}\right)-C\left(x^{2} x\right)=0, \quad \delta C\left(x x^{2} x\right)=x C\left(x^{2} x-x x^{2}\right)=0 .
$$

Gauge transformation

$$
\Delta C(x x)=2 x E(x)-E\left(x^{2}\right), \quad \Delta C\left(x x^{2}\right)=x E\left(x^{2}\right)+x^{2} E(x)=\Delta C\left(x^{2} x\right),
$$


By means of gauge transformations we can, for example, reduce $C(x x)$ to zero. Cohomology is the existence of the gauge invariant object $C\left(x x^{2}\right)+x C(x x)$ Mod $x^{2}$.

THEOREM 5.5. The space of the gange-equivalent evaluations, and the associated cohomology spaces on $Z_{p}(A, A)$ are as follows:

$$
\begin{aligned}
p=0: & A \\
& H^{0}(A, A)=\operatorname{span}\left\{1, x, \ldots, x^{n-1}\right\}, \text { dimension }=1 ; \\
p=1: & C(x) \\
& H^{1}(A, A)=\operatorname{span}\left\{x, \ldots, x^{n-1}\right\}, \text { dimension }=n-1 ; \\
p=2 k-1: & \sum_{l=0}^{n-2} x^{l} C\left(\phi^{\alpha-l}\right) \quad(k>1) \\
& H^{2 k-1}(A, A)=\operatorname{span}\left\{x, \ldots, x^{n-1}\right\}, \text { dimension }=n-1 ; \\
p=2 k: & \sum_{l=0}^{n-2} x^{l} C\left(\psi^{\alpha-l}\right) \quad \operatorname{Mod} \mathbb{C} x^{n-1} \\
& H^{2 k}(A, A)=\operatorname{span}\left\{1, x, \ldots, x^{n-2}\right\}, \text { dimension }=n-1 .
\end{aligned}
$$

Proof. By a direct and straightforward calculation we obtain, for $p=2 k$, $\sum_{l=0}^{n-2} x^{l} \Delta C\left(\psi^{\alpha-l}\right)=n x^{n-1} E(x \ldots x)$, and for $p=2 k-1, \quad \sum_{l=0}^{2} x^{l} \Delta C\left(\phi^{\alpha-1}\right)=0$.

PROPOSITION 5.6. The BGS "decomposition" for $k \geqslant 1$ is

$$
H_{2 k}=H_{2 k, k}, \quad H_{2 k+1}=H_{2 k+1, k+1} .
$$

Proof. The element $x \ldots x^{n-1} \in Z_{2 k}$ lifted to $Z_{2 k}(\mathbb{C}[x], \mathbb{C}[x])$, is

$$
\mathrm{d}\left(x x^{n-1}\right)^{k}=x^{n} \text { Sh }\left(x x^{n-1}\right)^{k-1} .
$$

If $\left(x x^{n-1}\right)^{k-1}$ is of type $H_{2 k-2, k-1}$, then the right-hand side is of type $H_{2 k-1, k}$, and $\left(x x^{n-1}\right)^{k}$ is of type $H_{2 k, k}$. Since $x x^{n-1}$ is indeed of type $H_{2,1}$ the result follows by induction. Similarly, $\mathrm{d}\left(x x^{n-1}\right)^{k} x=x^{n}(s h)\left(x x^{n-1}\right)^{k-1} x$, and the same argument applies mutatis mutandi.

\section{Appendix: Hochschild and Harrison Cohomology of Complete Intersections (by Maxim Kontsevich)}

I will explain here a way to calculate Hochschild and Harrison cohomology groups for algebras of functions on singular planar curves, etc., based on Koszul resolutions. This calculation is standard and definitely known to specialists. 


\section{A1. REMINDER ON COMPLETE INTERSECTIONS AND KOSZUL RESOLUTION}

Results of this section can be found e.g. in the classical textbook [19].

Suppose that we are given a system of polynomial equations (say, over the field of complex numbers $\mathbf{C}$, one can replace it by an arbitrary field):

$$
f_{1}\left(z_{1}, \ldots, z_{n}\right)=0, \ldots, f_{m}\left(z_{1}, \ldots, z_{n}\right)=0
$$

Denote by $A$ the quotient algebra $P /\left(f_{1}, \ldots, f_{m}\right)$ where $P$ denotes the ring of polynomials $\mathbf{C}\left[z_{1}, \ldots, z_{n}\right]$.

We say that we have a complete intersection if the dimension of the set of solutions of the system above is $n-m$. A sufficient condition for this is that $f_{1}, \ldots, f_{m}$ form a regular sequence in $P$, i.e. for any $k \leq n$ element $f_{k}$ is not a divisor of zero in the quotient of $P$ by the ideal generated by $f_{1}, \ldots, f_{k-1}$.

THEOREM 1. Assume (in the previous notations) the condition of the complete intersection. Let us consider $\mathbf{Z}_{\leq 0}$-graded supercommutative superalgebra

$$
\tilde{A}:=P \otimes \wedge\left(\left\{\alpha_{j}\right\}_{j=1, \ldots, m}\right)
$$

where subalgebra $P$ is in degree 0 and generators $\alpha_{j}$ are in degree -1 , endowed with differential

$$
\mathrm{d}_{\tilde{A}}:=\sum_{j} f_{j} \frac{\partial}{\partial \alpha_{j}} .
$$

Then cohomology of this differential is zero in negative degrees and isomorphic to $P /\left(f_{1}, \ldots, f_{m}\right)$ in degree 0 .

In the above theorem one can replace $P=\mathbf{C}\left[z_{1}, \ldots, z_{n}\right]$ by the algebra of functions on arbitrary smooth $n$-dimensonal affine algebraic variety. Complex $\left(\tilde{A}, d_{\tilde{A}}\right)$ is called the Koszul resolution of $A$. Slightly abusing notations we will write $\tilde{A}=$ $\mathbf{C}\left[z_{1}, \ldots, z_{n} ; \alpha_{1}, \ldots, \alpha_{m}\right]$ meaning that $\left(\alpha_{i}\right)$ are fermionic (odd) variables. Here and later, variables denoted by Latin (respectively Greek) letters are even (respectively odd).

\section{A2. GENERALITIES ON HOCHSCHILD AND HARRISON COHOMOLOGICAL COMPLEXES FOR DIFFERENTIAL GRADED ALGEBRAS}

In what follows all complexes will be $\mathbf{Z}$-graded with the differential of degree +1 . A morphism of complexes is called a quasi-isomorphism iff it induces an isomorphism of cohomology groups. A vector space can be considered as a complex concentrated in degree 0 and endowed with zero differential.

Definitions of homological and cohomological Hochschild complexes extend immediately to the case of differential graded algebras (dga), the same for Harrison (co)homological complexes in the graded commutative case. The underlying Z-graded space for the cohomological Hochschild complex for a dga $F$ with 
coeffcients in a differential graded bimodule $M$ is defined as the infinite product (in the category of $\mathbf{Z}$-graded spaces)

$$
C^{\bullet}(F, M):=\prod_{n \geqslant 0} \underline{\operatorname{Hom}}\left(F[1]^{\otimes n}, M\right)
$$

where Hom is inner Hom-space in tensor category of $\mathbf{Z}$-graded spaces,

$$
(\underline{\operatorname{Hom}}(U, V))^{k}:=\prod_{n \in \mathbf{Z}} \operatorname{Hom}\left(U^{n}, V^{n+k}\right)
$$

and $F[1]$ denotes the complex obtained from $F$ by the shift of the grading, $F[1]^{k}:=F^{k+1}$. The formula for the differential in $C^{\bullet}(F, M)$ is the sum of a superversion of the formula for the differential in an ordinary algebra (in degree 0 ), and a term arising from the differential in $F$ itself (see e.g. Section 5.3 from [18] for a similar case of the homological Hochschild complex).

LEMMA 1. If $\phi: \tilde{F} \rightarrow F$ is a quasi-isomorphism between two dga's, then the corresponding cohomological Hochschild complexes $C^{\bullet}(F, F)$ and $C^{\bullet}(\tilde{F}, \tilde{F})$ are quasiisomorphic.

Proof. An algebra $F$ can be considered as a differential graded bimodule over $\tilde{F}$ via the homomorphism $\phi: \tilde{F} \rightarrow F$. Let us consider three complexes and natural homomorphisms between them:

$$
C^{\bullet}(\tilde{F}, \tilde{F}) \rightarrow C^{\bullet}(\tilde{F}, F) \leftarrow C^{\bullet}(F, F)
$$

All three complexes carry complete decreasing filtrations with the associated quotients (and maps between them)

$$
\underline{\operatorname{Hom}}\left(\tilde{F}[1]^{\otimes k}, \tilde{F}\right) \rightarrow \underline{\operatorname{Hom}}\left(\tilde{F}[1]^{\otimes k}, F\right) \leftarrow \underline{\operatorname{Hom}}\left(F[1]^{\otimes k}, F\right)
$$

We see that associated quotients are quasi-isomorphic, and applying spectral sequences we conclude that $C^{\bullet}(\tilde{F}, \tilde{F})$ and $C^{\bullet}(F, F)$ are quasi-isomorphic.

For a graded supercommutative $F$ one can define the Hodge decomposition for Hochschild cochains, and Harrison cohomology in the same way as in the usual non-graded case. In the above lemma the quasi-isomorphism between Hochschild cohomology of the resolution and of algebra itself is manifestly compatible with the Hodge decomposition.

\section{A3. CALCULATION OF HOCHSCHILD AND HARRISON COHOMOLOGY FOR COMPLETE INTERSECTIONS}

The cohomological Hochschild-Kostant-Rosenberg theorem says that the Hochschild cohomology of the algebra $\mathcal{O}_{X}$ of functions on an algebraic affine manifold $X$ is 
the algebra $T_{X}^{\text {poly }}$ of polyvector fields on $X$. Moreover, there is a canonical quasiisomorphism $T_{X}^{\text {poly }} \rightarrow C^{\bullet}\left(\mathcal{O}_{X}, \mathcal{O}_{X}\right)$ mapping polyvector field $f v_{0} \wedge \cdots \wedge v_{n}$ where $f \in \mathcal{O}_{X},\left(v_{i}\right)_{i=1, n}$ are derivations of $\mathcal{O}_{X}$, to the polylinear operator

$$
a_{1} \otimes \cdots \otimes a_{n} \mapsto f \sum_{\sigma \in \Sigma_{n}} \operatorname{sign}(\sigma) \prod_{i} v_{\sigma(i)}\left(a_{i}\right)
$$

The super-version of this theorem is also true, e.g. for supermanifold $Y=\mathbf{C}^{n \mid m}$, we have $\mathcal{O}_{Y}=\mathbf{C}\left[z_{1}, \ldots, z_{n} ; \alpha_{1}, \ldots, \alpha_{m}\right]$ and its Hochschild cohomology is the algebra $T_{Y}^{\text {poly }}$ :

$$
\begin{aligned}
T_{Y}^{\text {poly }} & =\mathbf{C}\left[z_{1}, \ldots, z_{n} ; \eta_{1}, \ldots, \eta_{n} ; \alpha_{1}, \ldots, \alpha_{m} ; b_{1}, \ldots, b_{m}\right], \\
\operatorname{deg}\left(z_{i}\right) & =0 \quad \operatorname{deg}\left(\eta_{i}\right)=+1, \quad \operatorname{deg}\left(\alpha_{j}\right)=-1, \quad \operatorname{deg}\left(b_{j}\right)=+2
\end{aligned}
$$

Here, the new variables $\eta_{i}, b_{j}$ have the meaning of derivations $\partial / \partial z_{i}, \partial / \partial \alpha_{j}$. Strictly speaking, here we should consider not polynomials but formal power series with respect to variables $\eta_{i}, b_{j}$, but it gives the same result in the category of $\mathbf{Z}$-graded spaces because there are only finitely many monomials in $\eta_{i}, b_{j}$ in any given degree.

The dga $\tilde{A}$ is obtained from $\mathcal{O}_{Y}$ by "switching on" the differential $\mathrm{d}_{\tilde{A}}$. Here, we describe the corresponding HKR description of the Hochschild cohomology of $\tilde{A}$, and therefore of $H^{\bullet}(A, A)$ by Lemma 1 .

PROPOSITION 1. Complex $C^{\bullet}(\tilde{A}, \tilde{A})$ is quasi-isomorphic to $T:=T_{Y}^{\text {poly }}$ endowed with the differential

$$
\mathrm{d}_{T}:=\sum_{i, j} \frac{\partial f_{j}}{\partial z_{i}} b_{j} \frac{\partial}{\partial \eta_{i}}+\sum_{j} f_{j} \frac{\partial}{\partial \alpha_{j}}
$$

The Hodge grading is given by counting variables $\eta_{i}, b_{j}$.

Proof. The formula for $\mathrm{d}_{T}$ is just the formula for the Lie derivative of a polyvector field on $Y=\mathbf{C}^{n \mid m}$ with respect to the odd vector field $\mathrm{d}_{\tilde{A}}=\sum_{j} f_{j} \frac{\partial}{\partial \alpha_{j}}$. It is easy to see that the formulas from above give a homomorphism of complexes

$$
\chi:\left(T, \mathrm{~d}_{T}\right) \rightarrow C^{\bullet}(\tilde{A}, \tilde{A})
$$

We have to prove that it is a quasi-isomorphism. Let us introduce $\mathbf{Z}_{\geqslant 0}$-grading $\operatorname{deg}_{\alpha}$ on $\mathcal{O}_{Y}$ by the total number of variables $\alpha_{j}$ (incidentally, it coincides with minus the standard $\mathbf{Z}$-grading on $\mathcal{O}_{Y}$ ). A Hochschild cochain $\mathcal{O}_{Y}^{\otimes n} \rightarrow \mathcal{O}_{Y}$ is called homogeneous of $\operatorname{deg}_{\alpha}$ degree $N \in \mathbf{Z}$ if it is homogenous with respect to grading $\operatorname{deg}_{\alpha}$ of degree $N$. The whole Hochschild complex $C^{\bullet}\left(\mathcal{O}_{Y}, \mathcal{O}_{Y}\right)$ is the product over all $N \in \mathbf{Z}$ of subcomplexes consisting of $\operatorname{deg}_{\alpha}$ degree $N$ cochains. The Hochschild differential of algebra $\mathcal{O}_{Y}$ preserves the $\operatorname{deg}_{\alpha}$ grading. The correction to the differential coming from $\mathrm{d}_{\tilde{A}}$ decreases this grading by 1 . Finally, it is obvious that for a non-zero cochain its $\operatorname{deg}_{\alpha}$ is bounded from below (by $-m$ ). Therefore we 
have a convergent spectral sequence proving that $\chi$ is a quasi-isomorphism. The statement about the Hodge grading is obvious.

Now we introduce a smaller complex

$$
\tilde{T}:=A\left[\eta_{1}, \ldots, \eta_{n} ; b_{1}, \ldots, b_{m}\right], \mathrm{d}_{\tilde{T}}:=\sum_{i, j} \frac{\partial f_{j}}{\partial x_{i}} b_{j} \frac{\partial}{\partial \eta_{i}}
$$

where the variables have the same grading as before, $\operatorname{deg}\left(\eta_{i}\right)=+1, \operatorname{deg}\left(b_{j}\right)=+2$.

THEOREM 2. Under the previous assumptions the Hochschild cohomology of A is isomorphic to the cohomology of complex $\left(\tilde{T}, \mathrm{~d}_{\tilde{T}}\right)$. The Hodge grading is given by counting variables $\eta_{i}, b_{j}$.

Proof. The obvious map $\left(T_{F}, \mathrm{~d}_{T}\right) \rightarrow\left(\tilde{T}, \mathrm{~d}_{\tilde{T}}\right)$ induces a quasi-isomorphism on graded quotients for the filtration by the total number of variables $\eta_{i}$.

The conclusion for the only non-trivial Harrison cohomology are in degrees 1 and 2 and are given by kernel and cokernel of the map

$$
A^{n} \stackrel{\left(\partial f_{j} / \partial_{z_{i}}\right)}{\longrightarrow} A^{m}
$$

In particular, there is no obstruction for commutative deformations as $\mathrm{Harr}^{3}$ $(A)=0$. It is easy to see that a miniversal commutative deformation of $A$ is given by any deformation $\tilde{f}_{1}(z, t), \ldots, \tilde{f}_{m}(z, t)$ of polynomials $f_{1}(z), \ldots, f_{m}(z)$ depending on formal parameters $t_{1}, \ldots, t_{N}$ where $N=r k \operatorname{Harr}^{2}(A)$, such that vectors

$$
v_{k}:=\left(\frac{\partial \tilde{f}_{1}}{\partial t_{k}}{ }_{\mid t=0}, \ldots,{\frac{\partial \tilde{f}_{m}}{\partial t_{k}}}_{\mid t=0}\right), \quad k=1, \ldots, N
$$

form a basis in $\operatorname{Harr}^{2}(A)=A^{m} /\left(\frac{\partial f_{j}}{\partial z_{i}}\right) A^{n}$. The deformed algebra is

$$
\mathbf{C}\left[\left[t_{1}, \ldots, t_{N}\right]\right]\left[z_{1}, \ldots, z_{n}\right] / I
$$

where $I$ is the completion with respect to the topology on $\mathbf{C}\left[\left[t_{1}, \ldots, t_{N}\right]\right]$ associated with the maximal ideal, of the ideal generated by $\tilde{f}_{1}(z, t), \ldots, \tilde{f}_{m}(z, t)$.

In particular, if we have only one equation $f(z)=f_{1}(z)=0$ then $\operatorname{Harr}^{2}(A)$ is the quotient $\mathbf{C}\left[z_{1}, \ldots, z_{n}\right] /\left(f, \partial f / \partial z_{1}, \ldots, \partial f / \partial z_{n}\right)$.

In the case $n=2$ and $m=1$, Hochschild cohomology groups consists of an unstable part in lower degrees and 2-periodically repeated block

$$
A \stackrel{\left(\partial_{z_{1}} f_{1}, \partial_{z_{2}} f_{1}\right)}{\longrightarrow} A \oplus A \stackrel{\left(\partial_{z_{2}} f_{1},-\partial_{z_{1}} f_{1}\right)}{\longrightarrow} A
$$

Finally, for $n=m=1, A=\mathbf{C}[z] /\left(z^{k}\right)$ we have

$$
H^{0}(A, A)=A \simeq \mathbf{C}^{k}, H^{l}(A, A) \simeq \mathbf{C}^{k-1} \text { for } l=1,2, \ldots
$$




\section{A4. CALCULATION OF HOCHSCHILD A HOMOLOGY WITH COEFFICIENTS WITH THE DIAGONAL BIMODULE, FOR COMPLETE INTERSECTIONS}

Similarly, one can calculate Hochschild homology $H_{*}(A, A)$ for complete intersections. Here is the final result:

THEOREM 3. In previous notations and under the assumption of complete intersection the Hochschild homology $H_{*}(A, A)$ of $A$ is isomorphic to the cohomology of complex $\tilde{\Omega}:=A\left[\xi_{1}, \ldots, \xi_{n} ; a_{1}, \ldots, a_{m}\right]$ where degrees of variables are $\operatorname{deg}\left(\xi_{i}\right)=$ $-1, \operatorname{deg}\left(a_{j}\right)=-2$ endowed with the differential $\mathrm{d}_{\tilde{\Omega}}:=\sum_{i, j} \frac{\partial f_{j}}{\partial z_{i}} \xi_{i} \frac{\partial}{\partial a_{j}}$. The Hodge grading is given by counting variables $\xi_{i}, a_{j}$.

The proof is parallel to one for the cohomological complex. An example of this calculation for the case of truncated polynomial ring can be found in [18, exercise E.4.1.8, Proposition 5.4.15].

\section{References}

1. Barr, M.: Harrison homology, Hochschild homology and triples. J. Algebra 8, 314 $323(1968)$

2. Barr, M.: Cohomology of commutative algebras, Doctoral dissertation, University of Pennsylvania, PA, USA (1962)

3. Bayen, F., Fronsdal, C., Flato, M., Lichnerowicz, A., Sternheimer, D.: Quantum mechanics as a deformation of classical mechanics. Ann. Phys. 111, 61-110; 111-151 (1978)

4. Dito, G., Flato, M., Sternheimer, S., Takhtajan, L.: Deformation quantization and Nambu mechanics. Commun. Math. Phys. 183, 1-22 (1997)

5. Fedosov, B.V.: A simple geometrical construction of deformation quantization. J. Diff. Geom. 40, 213-238 (1994)

6. Fleury, P.J.: Splittings of Hochschild's complex for commutative algebras. Proc. Am. Math. Soc. 30, 405-323 (1971)

7. Fronsdal, C.: Some ideas about quantization. Rep. Math. Phys. 15, 111-145 (1978)

8. Fronsdal C.: Harrison cohomology and abelian deformation quantization on algebraic varieties. Deformation quantization (Strasbourg, 2001), IRMA Lectures in Mathematics and Theoretical Physics Vol. 1, pp. 149-161. de Gruyter, Berlin (2002)

9. Fronsdal, C.: Abelian deformations. Proceedings of the IXth International Conference on Symmetry Methods in Physics, Yerevan, July 2001

10. Garsia, A.M.: Combinatorics of the free Lie algebra and the symmetric group, Analysis, et cetera, Research Papers Published in Honor of Jürgen Moser's 60th Birthday, pp. 309-392. Academic, New York (1990)

11. Gerstenhaber, M.: On the deformations of rings and algebras. Ann. Math. 79, 59-103 (1964)

12. Gerstenhaber M.: Developments from Barr's thesis, presented at the celebration of M. Barr's $60^{\text {th }}$ birthday, 30 June 1998

13. Gerstenhaber, M., Schack, S.D.: A Hodge-type decomposition for commutative algebra cohomology. J. Pure Appl. Algebra 48, 229-247 (1987)

14. Harrison, D.K.: Commutative algebras and cohomology. Trans. Am. Math. Soc. 104, 191-204 (1962) 
15. Hochschild, G., Kostant, B., Rosenberg, A.: Differential forms on regular affine algebras. Trans. Am. Math. Soc. 102, 383-408 (1962)

16. Kontsevich M.: Deformation quantization of Poisson manifolds. Lett. Math. Phys. 66, 157-216 (2003) (q-alg/9709040)

17. Kontsevich, M.: Operads and motives in deformation quantization. Lett. Math. Phys. 48, 35-72 (1999)

18. Loday, J.-L.: Cyclic homology, Grundlehren der Mathematischen Wissenschaften, Vol. 301, xx+513 pp. Springer, Berlin (1998)

19. Matsumura, H.: Commutative algebra. Benjamin, New York (1970)

20. Vey, J.: Déformation du crochet de Poisson sur une variété symplectique. Comment. Math. Helv. 50, 421-454 (1975) 